\title{
Determination of Benzalkonium Chloride from Biocide Products: Selectivity Study ${ }^{\dagger}$
}

\author{
Georgeta Ramona Ivan *, Rusandica Stoica, Elena Radu, Luiza Capra and Mihaela Manolache \\ National Research \& Development for Chemistry and Petrochemistry ICECHIM, Department of Analysis, \\ Tests and Testings, 202 Splaiul Independentei, 6th district, 060021 Bucharest, Romania; \\ irusandica@yahoo.com (R.S.); nina.radu58@yahoo.ro (E.R.); luizacapra@yahoo.com (L.C.); \\ dir.calitate@icechim.ro (M.M.) \\ * Correspondence: georgeta.ratea@gmail.com \\ + Presented at the 15th International Symposium "Priorities of Chemistry for a Sustainable Development" \\ PRIOCHEM, Bucharest, Romania, 30th October-1st November 2019.
}

Published: 17 October 2019

Keywords: benzalkonium chloride; biocides; selectivity

Biocidal products are substances or mixtures of several substances, used to combat harmful organisms for human or animal health. Substances with antibacterial effects include quaternary ammonium salts, such as benzalkonium chloride (BAC), didecyl chloride (DDAC), etc. Benzalkonium chloride (BAC) is a compound of the class of quaternary ammonium compounds (QAC) used as a cationic surfactant in personal hygiene, cosmetic and skin disinfection products [1]. BAC is a mixture of alkylbenzyldimethylammonium chlorides $\left[\mathrm{C}_{6} \mathrm{H}_{5} \mathrm{CH}_{2} \mathrm{~N}\left(\mathrm{CH}_{3}\right)_{2} \mathrm{RCl}\right]$, in which $\mathrm{R}=$ $\mathrm{C}_{12}, \mathrm{C}_{14}, \mathrm{C}_{16}, \mathrm{C}_{18}$ are the main compounds, and the antibacterial effects being given by the alkyl chain length and the quaternary ammonium groups [1,2].

In this study we compare two methods of analysis for quantitative determination: chemical titration [3] and liquid chromatography (HPLC-UV) [4]. Chemical titration is an iodometric method in a strongly acidic medium, in which the $0.05 \mathrm{M}$ potassium iodate solution is used as titrant (titration with extracted end point) [3]. HPLC determinations were performed using an Agilent 1100 Series HPLC, equipped with a binary pump, a degasser, an autosampler and a spectrophotometric detector in UV-Vis, and Agilent Chemstation software was used for data acquisition and analysis. Chromatographic parameters were injection volume, $10 \mu \mathrm{L}$; analysis time, $25 \mathrm{~min}$; a Zorbax Eclipse XDB-C18 column $(5 \mu \mathrm{m}, 4.6 \mathrm{~mm} \times 150 \mathrm{~mm})$; and flow rate, $1.5 \mathrm{~mL} / \mathrm{min}$, isocratic conditions 20:80 A:B $(\mathrm{v} / \mathrm{v})$, where $\mathrm{A}=0.4 \mathrm{M} / \mathrm{L} \mathrm{NaCl}$ solution and $\mathrm{B}=$ methanol [4]. $\mathrm{BAC}$ monitoring was performed at a wavelength of $210 \mathrm{~nm}$ with the help of a UV-Vis spectrophotometric detector. The identification was performed by comparing the retention time of the analyzed compound with the retention time of the standard solution. Confirmation of the presence of BAC alkylic homologues, prior to quantitative determination, was performed using the mass spectrometry detection technique with a time-of-flight detector (TOF LC/MS).

As a measure of selectivity, for calculating the degree of recovery, the standard method of addition was applied. As a measure of selectivity, the degree of recovery determined by the standard addition method was used. For the titrimetric method we studied the influence of the presence of possible interferences different from those of the QAC class, such as ethyl and isopropyl alcohols, glutaraldehyde, non-ionic surfactants, stabilizers, etc. Regarding the HPLC-UV method, we were particularly interested in the selectivity with respect to other quaternary ammonium salts, such as didecyldimethylammonium chloride (DDAC) and cationic surfactants. 
The data obtained demonstrate the selectivity of the titrimetric method in relation to the following compounds: ethyl and isopropyl alcohols, glutaraldehyde, non-ionic surfactants, stabilizers, etc. The HPLC-UV method is additionally selective in relation to other quaternary ammonium compounds, such as DDAC and cationic surfactants. The titrimetric method proves to be usable for biocidal products on the market that contain only a single quaternary ammonium salt in the presence of the other compounds studied, while the chromatographic method is specific only for the benzalkonium chloride contained by the biocidal products in the co-presence of other quaternary ammonium salts and other classes of compounds.

\section{References}

1. Liu, J.; Deng, W.; Yu, M.; Wen, R.; Yao, S.; Chen, B., Rapid determination of benzalkonium chloride using paper spray mass spectrometry. J. Pharmaceut. Biomed. 2017, 145, 151-157.

2. el Hage, S.; Lajoie, B.; Stigliani, Je.; Furiga-Chusseau, A.; Roques, C.; Baziard, G., Synthesis, antimicrobial activity and physico-chemical properties of somen-alkyldimethylbenzylammonium halides. J. Appl. Biomed. 2014, 12, 245-253.

3. European Pharmacopoeia 8.8, 04/2009:0371-Benzalkonium Chloride Solution, 1638-1640.

4. Nakamura, K.; Morikawa, Y.; Matsumoto, I., Rapid analysis of ionic and nonionic surfactant homologs by performance liquid chromatography. JAOCS 1981, 58, 72-77.

() 2019 by the authors. Licensee MDPI, Basel, Switzerland. This article is an open access article distributed under the terms and conditions of the Creative Commons Attribution (CC BY) license (http://creativecommons.org/licenses/by/4.0/). 